\title{
A General Model for Representing Arbitrary Unsymmetries in Various Types of Network Analysis
}

Rønne-Hansen, Jan

Published in:

IEEE Transactions on Power Systems

Link to article, DOI:

$10.1109 / 59.630477$

Publication date:

1997

Document Version

Publisher's PDF, also known as Version of record

Link back to DTU Orbit

Citation (APA):

Rønne-Hansen, J. (1997). A General Model for Representing Arbitrary Unsymmetries in Various Types of Network Analysis. IEEE Transactions on Power Systems, 12(1), 1323-1331. https://doi.org/10.1109/59.630477

\section{General rights}

Copyright and moral rights for the publications made accessible in the public portal are retained by the authors and/or other copyright owners and it is a condition of accessing publications that users recognise and abide by the legal requirements associated with these rights.

- Users may download and print one copy of any publication from the public portal for the purpose of private study or research.

- You may not further distribute the material or use it for any profit-making activity or commercial gain

- You may freely distribute the URL identifying the publication in the public portal

If you believe that this document breaches copyright please contact us providing details, and we will remove access to the work immediately and investigate your claim. 


\title{
A General Model for Representing Arbitrary Unsymmetries in Various Types of Network Analysis.
}

\author{
By prof., Ph.D. J. Rønne-Hansen, Electric Power Engineering Department, \\ Technical University of Denmark
}

\begin{abstract}
When dealing with unsymmetric faults various proposals have been put forward. In general they have been characterized by specific treatment of the single fault in accordance with the structure and impedances involved [1]. The model presented here is based on node equations and was oniginally developed for transient stability studies in order to allow for an arbitrary fault representation as seen from the positive sequence network. The method results in impedances - or admittances - combining the negative sequence and zero sequence representation for the symmetrical network with the structure and electrical constants of the unsymmetry involving one or more buses. These impedances are introduced in the positive sequence network in the nodes involved in the unsymmetrical conditions. In addition, the model can be used for static fault current analysis and presents also in this connection a general method of solution; the method is easy to use since the task of user is limited to establishing the node admittance matrix of the unsymmetry expressed in phase coordinates. The paper is completed with two examples showing the application of the method, one fault for which a well known representation exists and one complicated fault situation which has not been treated before for traditional transient stability analysis.
\end{abstract}

Keywonds - Power system stability, unsymmetric conditions.

\section{INTRODUCTION}

The problem of representing unsymmetrical faults in a positive sequence network was attacked as soon as the symmetrical components were introduced in stability analysis. Since Kimbark in 1946 [2] presented the treatment of a dozen of the most common faults by a separate and tedious formation of the necessary equations for each single fault. This method has been used in each textbook ever since (see e.g. [1] and most recently [4]). Although Kron in 1939[3] showed

96 SM 568-6 PWRS A paper recommended and approved by the IEEE Power System Engineering Committee of the IEEE Power Engineering Society for presentation at the 1996 IEEE/PES Summer Meeting, July 28 - August 1, 1996, in Denver, Colorado. Manuscript submitted July 25, 1995; made available for printing June 27, 1996. how mesh equations can be generated automatically for shunt faults, the transition to node equations isolating the boundary between the positive sequence representation and the rest of the system has not been carried through. An attempt by Wedin [8] to solve the problem did not result in a useful method. Therefore, the handling of e.g. combined series and shunt faults, shunt faults involving more than one node, or simultaneous faults has been carried out in a full three phase representation of the entire network.

The representation of any case - how complicated it might be - seen from the positive sequence network is presented here. This solution allows the determination of any voltage and current in the fault and its connections to the network. Since the positive sequence representation is coupled to the rest of the system by means of a transformer winding, the voltage over and the current through this winding mirrors the joint influence of all the systems behind this winding. Thus, the impedance which should be added instead of the winding in the positive sequence network is determined.

\section{A NOTE ON TERMINOLOGY}

In this paper the word "bus" is used for a junction in the network (power station, substation etc.). "Node" on the contrary is used internally in a system consisting of three four-winding phase-shifters coupling together the three symmetric component representations and the three phases representing one bus. To the six accessible nodes the 12 windings are further interconnected by means of six internal nodes (see fig. 1A in the appendix).

\section{THE SYSTEM OF PHASE-SHIFTERS}

Any unsymmetry in a generally symmetrical network can easily be represented in a three phase representation. When the network must be represented by means of symmetrical components, the unsymmetry, however, results in a coupling between the three during normal operation independent schemes.

Such couplings can be represented by means of transformers in the general case phase-shifters. In fig. 1 a combination of three four-winding transformers connects the three phases (a; $\mathrm{b}$ and $\mathrm{c}$ ) with the three impedances representing the symmetrical components $(\mathrm{P} ; \mathrm{N}$ and $\mathrm{Z}$ ). Only the positive sequence representation is activated by a source. The nodes $\mathrm{a}, \mathrm{b}$ and $\mathrm{c}$ plus ground are available for the representation of any unsymmetric coupling. 
Unfortunately, the transformers need a leakage inductance in order to form the node equations combining currents and voltages. This inductance, of course, is a pure computational necessity and must be chosen small enough to prevent inaccuracy in the results, but sufficiently large to prevent numerical problems. For the sake of simplicity the same admittance, $Y_{t}$, is chosen for all the transformers.

In the appendix the node admittance matrix for the system is developed including all the nodes. First, however, the constraint matrix for the actual transformers is established together with the node incidence matrix. From the full node admittance matrix (A4) the $\bar{Y}_{a d}$ shown below, eq. (1), is calculated using traditional matrix reduction preserving the six external nodes.

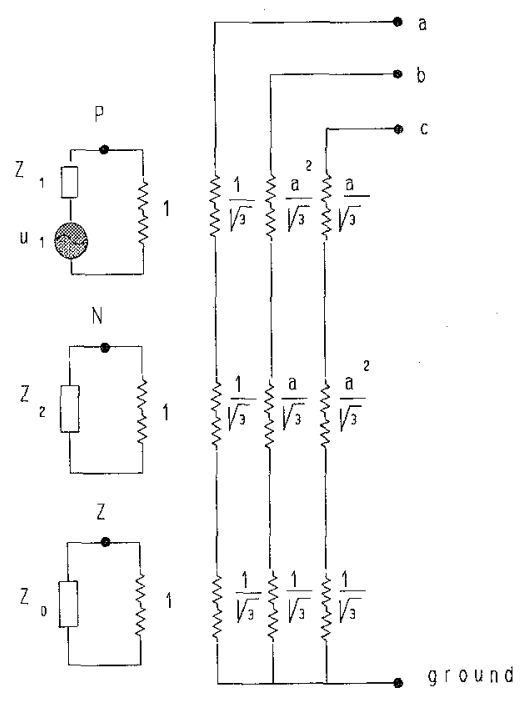

Fig. 1 The "adapter"

$$
\bar{Y}_{a d}=\frac{Y_{t}}{3} \cdot\left[\begin{array}{cccccc}
P & N & Z & a & b & c \\
1 & 0 & 0 & -\frac{1}{\sqrt{3}} & -\frac{1}{\sqrt{3}} a & -\frac{1}{\sqrt{3}} a^{2} \\
0 & 1 & 0 & -\frac{1}{\sqrt{3}} & -\frac{1}{\sqrt{3}} a^{2} & -\frac{1}{\sqrt{3}} a \\
0 & 0 & 1 & -\frac{1}{\sqrt{3}} & -\frac{1}{\sqrt{3}} & -\frac{1}{\sqrt{3}} \\
-\frac{1}{\sqrt{3}} & -\frac{1}{\sqrt{3}} & -\frac{1}{\sqrt{3}} & 1 & 0 & 0 \\
-\frac{1}{\sqrt{3}} a^{2} & -\frac{1}{\sqrt{3}} a & -\frac{1}{\sqrt{3}} & 0 & 1 & 0 \\
-\frac{1}{\sqrt{3}} a & -\frac{1}{\sqrt{3}} a^{2} & -\frac{1}{\sqrt{3}} & 0 & 0 & 1
\end{array}\right]^{P}
$$

As usual $\mathrm{a}=1 \angle 120^{\circ}$.
The development of this node admittance matrix demands the formulation of a specific turns ratio matrix based on the constant voltage/winding relation as described in [4]. This technique of classification of constraints dealing with each class separately was introduced in [3].

The node admittance matrix $\bar{Y}_{a d}$, eq. (1), must be completed by adding the symmetrical component representation of the network in the three first diagonal elements, $\bar{Y}_{n e t}$, and the admittances for the fault in the rows/columns corresponding to the phase quantities $\bar{Y}_{\text {fault }}$ i.e.

$$
\bar{Y}_{\text {tot }}=\bar{Y}_{a d}+\left(\begin{array}{cc}
\bar{Y}_{\text {net }} & 0 \\
0 & \bar{Y}_{\text {fault }}
\end{array}\right)
$$

$\vec{Y}_{\text {fault }}$ can be illustrated by an example: consider a double line-to-ground fault in phase $a$ and $c$ as indicated in fig. 2 .

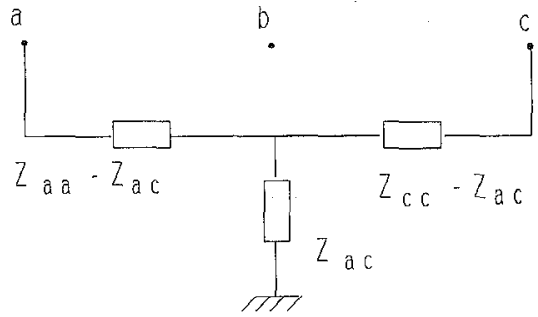

Fig. 2. Double Line-to-ground with the necessary impedances.

Note, that since admittances must be used, only $Z_{\mathrm{ac}}$ can be zero, whereas $Z_{a 2}$ and $Z_{c c}$ both must represent some small quantity in order to ensure formation of the node admittance matrix

$$
\bar{Y}_{f a u l t}=\left(\begin{array}{ccc}
Y_{a a} & 0 & Y_{a c} \\
0 & 0 & 0 \\
Y_{a c} & 0 & Y_{c c}
\end{array}\right)
$$

where

$$
\left(\begin{array}{cc}
Y_{a a} & Y_{a c} \\
Y_{a c} & Y_{c c}
\end{array}\right)=\left(\begin{array}{ll}
Z_{a a} & Z_{a c} \\
Z_{a c} & Y_{c c}
\end{array}\right)^{-1}
$$


When inverting $\bar{Y}_{\text {tot }}$ from (2) to form the node impedance matrix, $\bar{Z}_{\text {tot }}$, the Thevenin voltage source in the positive sequence network is the only source. Emphasizing the positive sequence network, fig. 3 , reveals that seen from node $P$ the remaining part of the network plus the fault are described by means of a single impedance. In case of a line-to-ground fault this impedance is well known as

$$
\mathrm{Z}=\mathrm{Z}_{2}+\mathrm{Z}_{0}+3 \cdot \mathrm{Z}_{\text {fault }}
$$

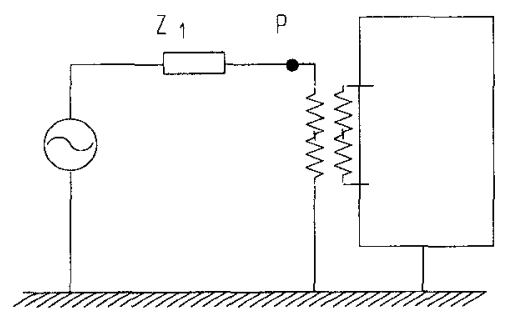

Fig. 3. Positive sequence representation

Here, node $\mathrm{P}$ corresponds with the first row/column in the node admittance and impedance matrix. Thus, when leaving out the positive sequence representation from the node admittance matrix, the inverse matrix corresponding to node $P$ (position (1,1)) will give the correct impedance representing the foult and the remaining data for the network. A primitive example is shown in section 5 .

\section{SIMULTANEOUS FAULTS AND FAULTS INVOLVING MORE THAN ONE BUS IN THE NETWORK.}

When dealing with simultaneous faults or faults involving more than one bus (e.g. both ends of a transmission line) one system of phase-shifters is needed for each bus involved. The necessary number of matrices are arranged along the diagonal in a matrix with $6 \cdot \mathrm{n}$ rows/columns where $\mathrm{n}$ is the number of buses involved.

For two buses, $i$ and $j$, the structure of the node admittance matrix is:

$$
\bar{Y}_{\text {tot }}=i_{j}\left(\begin{array}{cc}
i & j \\
\bar{Y}_{a d} & \overline{0} \\
\overline{0} & \bar{Y}_{a d}
\end{array}\right)+\left(\begin{array}{cccc}
i & j \\
\bar{Y}_{N i i} & \overline{0} & \bar{Y}_{N i j} & \overline{0} \\
\overline{0} & \bar{Y}_{F i} & \overline{0} & \bar{Y}_{F i j} \\
\bar{Y}_{N i j} & \overline{0} & \bar{Y}_{N i j} & \overline{0} \\
\overline{0} & \bar{Y}_{F U} & \overline{0} & \bar{Y}_{F i j}
\end{array}\right) j
$$

Index $\mathrm{N}$ refers to the network (symmetrical components) and index $\mathrm{F}$ to the fault(s). If the unsymmetries in the two buses are not directly connected (independent simultaneous faults)

$$
\bar{Y}_{F i j}=\overline{0} \text {. }
$$

In this specific case again the representation of the influence of the unsymmetries towards the positive sequence network is determined by

- leaving out the positive sequence data in $\bar{Y}_{\text {tot }}$

- inverting $\bar{Y}_{\text {tot }}$ to form $\bar{Z}_{\text {tot }}$

- extracting the positive sequence data from $\bar{Z}_{t o t}$ (i.e. the elements $(1,1) ;(1,7) ;(7,1)$ and $(7,7))$.

If the node impedance matrix does not exist, a reduction of $\bar{Y}_{\text {tot }}$ to represent the node admittance matrix, $\bar{Y}_{\text {tot }}$, treating the positive sequence nodes only, is required. Consequently, in this case again a $2 \times 2$ matrix.

\section{TWO EXAMPLES}

First, a simple example is chosen for which the result is well known.

For a bus in a network the short circuit capacity is 1250MVA at $100 \mathrm{kV}$. The negative sequence and the positive sequence representations are equal and purely inductive.

For the zero sequence one impedance $Z_{0}=j 20$ is used. A single line-to-ground fault occurs in phase $b$ involving the fault impedance $15+\mathrm{j} 0 \Omega$.

In this specific case the matrix which must be added to $\vec{Y}_{a d}$ is

$$
\left(\begin{array}{cccccc}
0 & 0 & 0 & 0 & 0 & 0 \\
0 & -j .125 & 0 & 0 & 0 & 0 \\
0 & 0 & -j .05 & 0 & 0 & 0 \\
0 & 0 & 0 & 0 & 0 & 0 \\
0 & 0 & 0 & 0 & .0667 & 0 \\
0 & 0 & 0 & 0 & 0 & 0
\end{array}\right)
$$

Selecting $Y_{t}=-j 300 \mathrm{~S}$ for the phase-shifter windings gives the following $\bar{Y}_{\text {tot }}$ adding (1) and (6) 


$$
\left(\begin{array}{cccccc}
-j 100 & 0 & 0 & j \frac{100}{\sqrt{3}} & -50-j \frac{50}{\sqrt{3}} & 50-j \frac{50}{\sqrt{3}} \\
0 & -j 100,125 & 0 & j \frac{100}{\sqrt{3}} & 50-j \frac{50}{\sqrt{3}} & -50-j \frac{50}{\sqrt{3}} \\
0 & 0 & -j 100,05 & j \frac{100}{\sqrt{3}} & j \frac{100}{\sqrt{3}} & j \frac{100}{\sqrt{3}} \\
j \frac{100}{\sqrt{3}} & j \frac{100}{\sqrt{3}} & j \frac{100}{\sqrt{3}} & -j 100 & 0 & 0 \\
50-j \frac{50}{\sqrt{3}} & -50-j \frac{50}{\sqrt{3}} & j \frac{100}{\sqrt{3}} & 0 & .0667-j 100 & 0 \\
-50-j \frac{50}{\sqrt{3}} & 50-j \frac{50}{\sqrt{3}} & j \frac{100}{\sqrt{3}} & 0 & 0 & -j 100
\end{array}\right)
$$

Inverting this matrix gives in position $(1,1)$

$$
\underline{Z}=44,97+\mathrm{j} 28,05 \Omega
$$

where the correct value is $Z=Z_{2}+Z_{0}+3 \cdot Z_{\text {fault }}=45+j 28 \Omega$. So, the error is less than $2 \%$.

The second example deals with a more complicated case in which the two ends of a transmission line have been coupled in different ways as indicated in fig. 4 .

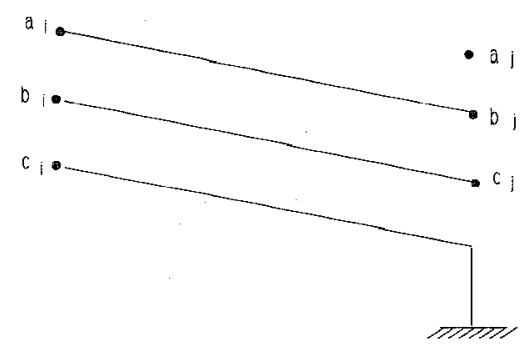

Fig. 4 Falsely connected line

For the line the following data are known:

$$
Z_{1}=Z_{2}=4+j 40 \Omega \text { and } Z_{0}=7+j 52 \Omega
$$

In phase coordinates the self-impedance of each conductor becomes $Z_{\mathrm{e}}=5+\mathrm{j} 44 \Omega$ and the mutual impedance $\mathrm{Z}_{\mathrm{m}}=1+\mathrm{j} 4 \Omega$.

By inversion of the phase coordinate impedance matrix, the following admittances are found.

$Y_{e}=.0025-j .0228 S$ and $Y_{m}=-2 \cdot 10^{-5}+j .0020 S \cong j .0020 S$

Here, the admittance matrix is defined as

$$
\bar{Y}_{l}=\left(\begin{array}{lll}
Y_{e} & Y_{m} & Y_{m} \\
Y_{m} & Y_{e} & Y_{m} \\
Y_{m} & Y_{m} & Y_{e}
\end{array}\right)
$$

Hence, the odd signs in the mutual admittance.

Forming the node admittance matrix in this case demands application of the node incidence matrix, $\bar{A}$, and forming the triple product $\bar{A}^{t} \cdot \bar{Y}_{l} \cdot \bar{A}$ The orientation of the line is chosen from $\mathrm{i}$ towards $\mathrm{j}$. Then

$$
\bar{A}=\left(\begin{array}{cccccc}
a_{i} & b_{i} & c_{i} & a_{j} & b_{j} & c_{j} \\
1 & & & & -1 & \\
& 1 & & & & -1 \\
& & 1 & & &
\end{array}\right)
$$

and the node admittance matrix becomes

$$
\bar{A}^{t} \bar{Y}_{l} \bar{A}=\left(\begin{array}{cccccc}
Y_{e} & Y_{m} & Y_{m} & 0 & -Y_{e} & -Y_{m} \\
Y_{m} & Y_{e} & Y_{m} & 0 & -Y_{m} & -Y_{e} \\
Y_{m} & Y_{m} & Y_{e} & 0 & -Y_{m} & -Y_{m} \\
0 & 0 & 0 & 0 & 0 & 0 \\
-Y_{e} & -Y_{m} & -Y_{m} & 0 & Y_{e} & Y_{m} \\
-Y_{m} & -Y_{e} & -Y_{m} & 0 & Y_{m} & Y_{e}
\end{array}\right)=\left(\begin{array}{ll}
\bar{Y}_{F i i} & \bar{Y}_{F i j} \\
\bar{Y}_{F j i} & \bar{Y}_{F i j}
\end{array}\right)
$$

$Y_{e}$ and $Y_{m}$ are described above.

For the network the following data are available:

$$
\bar{Z}_{1}=\bar{Z}_{2}={ }_{j}^{i}\left(\begin{array}{cc}
j 8 & j 3.7 \\
j 3.7 & j 13.5
\end{array}\right) \Omega \text { and } Z_{0}={ }_{j}^{i}\left(\begin{array}{cc}
j 20 & j 8 \\
j 8 & j 26
\end{array}\right) \Omega
$$

giving the admittances:

$$
\bar{Y}_{2}={ }_{j}^{i}\left(\begin{array}{ll}
-j .1431 & j .0392 \\
j .0392 & -j .0848
\end{array}\right) S \text { and } \bar{Y}_{0}={ }_{j}^{i}\left(\begin{array}{ll}
-j .0570 & j .0175 \\
j .0175 & -j .0439
\end{array}\right) S
$$

Referring to eq. (5)

$\bar{Y}_{N I i}=\left(\begin{array}{lll}0 & & \\ & -j .1431 & \\ & & -j .0570\end{array}\right) \quad \bar{Y}_{N i j}=\bar{Y}_{N i i}=\left(\begin{array}{lll}0 & & \\ & j .0392 & \\ & & j .0175\end{array}\right)$ and

$$
\bar{Y}_{N i j}=\left(\begin{array}{lll}
0 & & \\
& -j .0848 & \\
& & -j .0439
\end{array}\right)
$$


Inverting the $12 \times 12$ matrix, $\bar{Y}_{\text {tot }}$, gives the following positive sequence representation of the fault:

$$
\bar{Z}_{1}=\left(\begin{array}{cc}
14.40+j 157.92 & 143.49-j 98.78 \\
-159.04-j 71.78 & 23.33+j 265.48
\end{array}\right) \Omega
$$

In order to check this result the following independent and exact procedure was chosen:

- The network data were converted to phase quantities and the "fault" introduced in this three-phase representation (node impedance matrix).

- The unsymmetrical node impedance matrix was transformed to symmetrical components.

- The positive sequence part of this matrix was isolated and inverted to form the positive sequence node admittance matrix.

- The positive sequence node admittance matrix for the symmetrical network was subtracted from this matrix and the remains inverted.

The result is:

$$
\left(\begin{array}{cc}
14.52+j 158.60 & 144.34-j 99.48 \\
-160.08-j 72.15 & 23.63+j 267.18
\end{array}\right) \Omega
$$

The difference in result between the two totally independent methods is less than $1 \%$.

Although the three-phase algorithm used to test the results found by means of the phase-shifters may appear simpler, this method is preferred due to the following reasons:

- it is easy to form the node admittance matrix for the unsymmetry,

- a node impedance representation does not necessarily exist in the specific case,

- the inclusion and finally subtraction of the positive sequence network is a source of inaccuracy.

\section{A PRELIMINARY REMARK ON ACCURACY}

The small leakage inductances necessitated by the phaseshifters, natural are a source of numerical error. When choosing the inductance small $\left(Y_{t}=-j 300 S\right)$, the two examples above show very slight influence on the results. An attempt to change the inductance by a factor 3 up and down gave slight variations in data, but no dramatic deviations from the correct results were found. In general, an accuracy in the range of a few per cent was obtained. This must be considered satisfactory since it is not much inferior to the general accuracy for many network data. And the conditions in a fault are neither well known nor ideal! How the existence of negative resistance affects numerical properties during the integration is under investigation; anyway the representation of phase-shifters most often will involve such phenomena inside the balanced network.

\section{CONCLUSION}

The method outlined in this paper, with sufficient accuracy allows the inclusion of any unsymmetrical condition involving one or more nodes to be represented in a positive sequence representation. Consequently, the influence of such conditions in the positive sequence scheme can be simulated accurately. As a posibility of pure accademical interest, the same procedure can be used for the negative or zero sequence scheme as well! Finally, the method allows representation of faults at any distance from the substations to be thoroughly investigated without representing the faulted position itself in the positive sequence scheme.

Therefore, many cases which previously demanded full three phase representation can now be accurately dealt with in a much less expensive manner.

\section{APPENDIX}

A full description of the procedure is given in [4] but will be resumed below.

The principle in dealing with transformer banks is to formulate the constraints from the turns ratios (including phase shift) in a separate matrix, $N_{0}$, helping to formulate the node admittance matrix together with the node incidence matrix, $\bar{A}$ (see [4]).

The matrix $\bar{N}_{0}$, referring to node (and cut-set) equations, simply states the interrelationship between the voltages determined by the coils. Except for one reference coil in each transformer, a leakage inductance must exist in each winding. This inductance is a fictitious quantity which, concequently, can be assigned the same value for all coils since the only function is to secure the necessary relationship between voltages and currents. Referring again to [3] the node admittance matrix can be calculated as

$$
\bar{Y}_{N}=\bar{A}^{t} \cdot \bar{N}_{0}^{t *} \cdot \bar{Y}_{0} \cdot \bar{N}_{0} \cdot \vec{A}
$$


Here, $\bar{Y}_{t}$ must contain one admittance per coil excluding the reference coils. Fig. A1 shows the necessary twelve nodes and the leakage admittance which is chosen identically for the nine coils and designated $Y_{t}$. As reference coils are chosen those in the positive, the negative, and the zero sequence schemes.

For the transformer connecting the positive sequence scheme to the phase quantities the following relations exist:

$$
u_{1}=\sqrt{3} u_{4}=\frac{\sqrt{3}}{a^{2}} u_{7}=\frac{\sqrt{3}}{a} u_{10}
$$

giving rise to the three relationships

$$
\frac{1}{\sqrt{3}} u_{1}-u_{4}=0 ; \quad \frac{a^{2}}{\sqrt{3}} u_{1}-u_{7}=0 \text { and } \frac{a}{\sqrt{3}} u_{1}-u_{10}=0
$$

Similar relationships exist for the negative sequence and zero sequence couplings.

The following $\bar{N}_{0}$ matrix emerges:

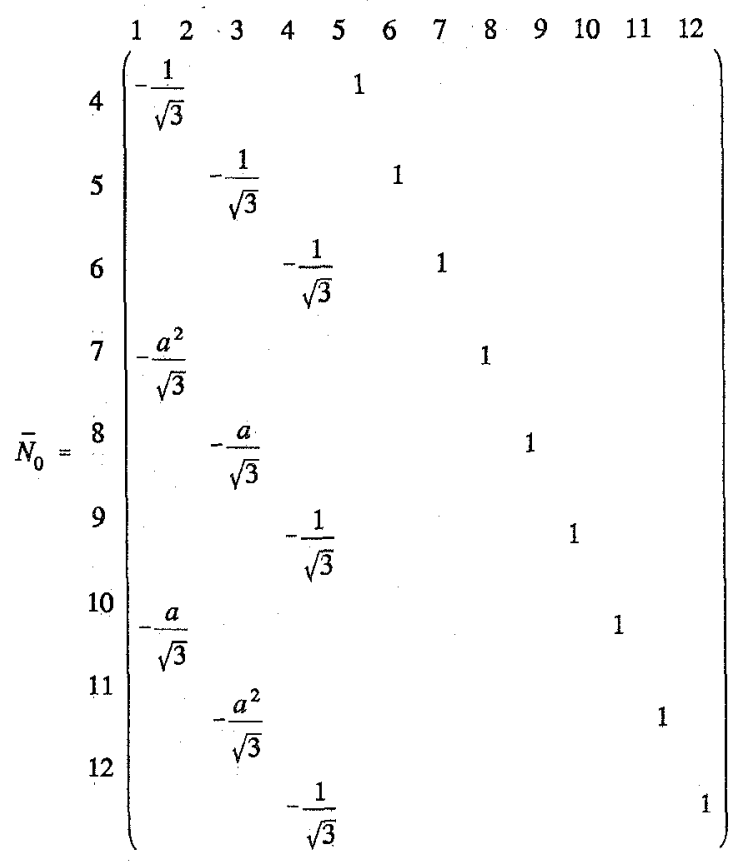

The node incidence matrix is

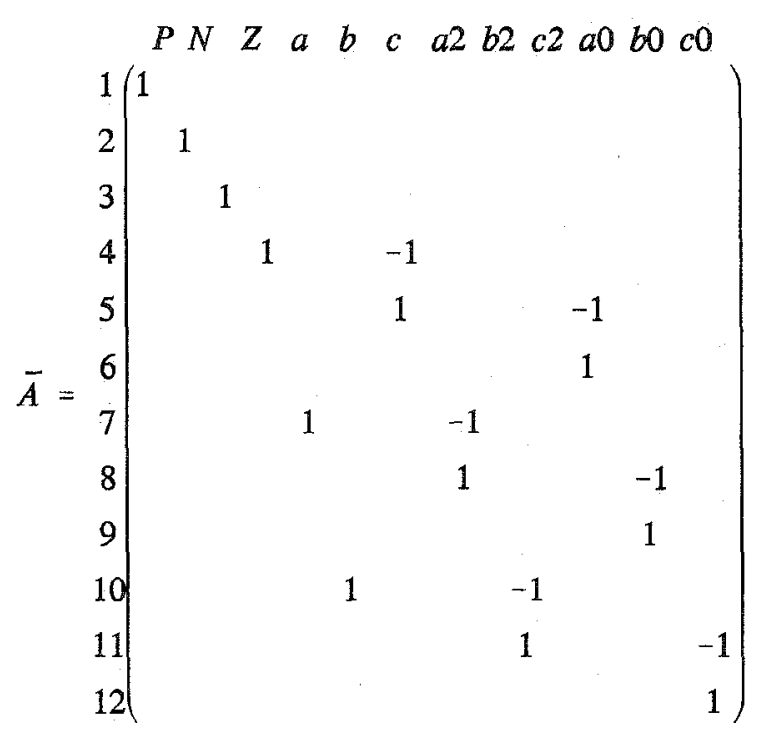

For the matrix $\vec{Y}_{0}$ the simple form Kronecker's $\Delta$ of the order nine times $Y_{t}$ is used.

Applying these matrices to (A1) gives the complete node admittance matrix for the phase-shifters, i.e.

$\bar{A}^{t} \bar{N}_{0}^{t *} \bar{Y}_{0} \bar{N}_{0} \bar{A}=$

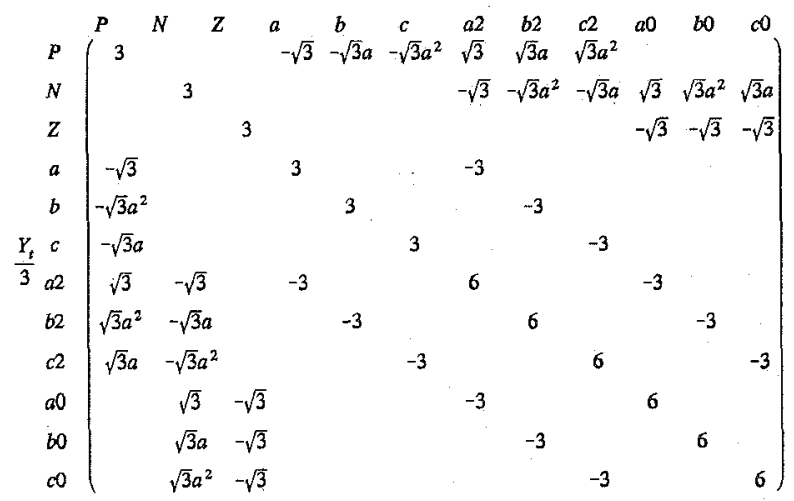

(1) appears by removing the 6 last nodes from (A4) by means of matrix reduction.

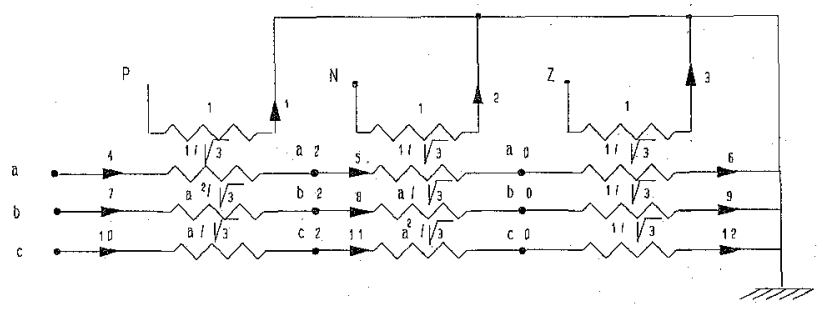

Fig. A1

The system of phase-shifters for one bus. Branches oriented and numbered. Turns ratios (complex numbers) are specified. 
For the sake of simplicity, the leakage succeptances are not displayed. $Y_{t}$ is coupled in parallel with the windings in the nine branches connected between the phase nodes and ground.

\section{REFERENCES}

1. Anderson, P: Analysis of Faulted Power Systems, The Iowa State University Press/Ames, 1973

2. Kimbark, E.W.: Power System Stability II, John Wiley 1948

3. Kron, G.: Tensor Analysis of Networks, Mac Donald, 1939

4. Kundur, Prabhar Power System Stability and Control, Mc Grow Hill Inc. 1994

5. J. Richard Honsen: A Systematic Approach to Power System Analysis, Ph.D. thesis, Techn. Univ. of Denmark, 1967

6. J. Richard Hansen: The General Constraint Matrices for Transformer Turns Ratios, 6th Power System Computation Conference, Secheim, Germany 1978.

7. J. Ronne-Honsen: Representing an Arbitrary Fault in a Positive Sequence Programme, Internal Report. Electric Power Engineering Dept., Tech. Univ. of Denmark, 1995 (23 pages).

8. Kurt Wedin: Matrismetodik för studier av driftfrekventa förhållanden $\mathrm{i}$ komplexa nät (in swedish). [Matrix method for studying fundamental frequency conditions in complex networks]. Royal Institute of Technology, Stockholm, 1965.

\section{BIOGRAPHY}

J. Ronne-Hansen was born in Ronne, Bornholm, August 9th 1936. He received his Msc EE from the Technical University of Denmark in 1962 and in 1967 Ph.D. from the same university. In the period 1962-1977 he was employed with the NESA Power Co., Copenhagen, and 1977-1980 with the Association of Dansh Power Undertakings, Copenhagen. In 1980 he became full professor of electric power engineering at the Technical University of Denmark. 


\section{Discussion}

M. Akke (Sydkraft Aktiebolag, Carl Gustavs väg 1, S-205 09 Malmö, Sweden): The author is congratulated to an interesting paper. Is it possible that the proposed method can be used to do "hybrid-simulation" where parts of the network have a full three-phase representation and other parts of the network are only represented by the positive sequence component?

One interesting application is dynamic simulation to determine the critical clearing time for faults that have a negative sequence component. Could a combined network model be used, where equipment close to the fault have a three phase representation and parts remote from the fault are only represented with the positive sequence?

Manuscript received August 20, 1996.

Göran Andersson (Royal Institute of Technology, S-100 44 Stockholm, Sweden.):

The author is commended for an interesting and well written paper that seems to open a number of new possibilities in power system analysis. I want to make some comments and put some questions and the author's response on these would be appreciated.

1. One reason to use a more sophisticated analytical approach rather than "brut force" methods employing extensive use of computers would be to gain more insight into the problem, sometimes by sacrificing some accuracy. Is this statement applicable for the presented method, and if yes, what are the additional insights gained?

2. In the abstract of the paper it is mentioned that the method can be used to enhance the modelling of unsymmetrical conditions in transient stability studies. However, this issue is not further elaborated in the paper. It would be of interest to know how the author envisages that this method should be incorporated in a transient stability programme and what are the main improvements as compared with the methods currently used in such programmes.

Manuscript received August 22, 1996.

\section{J. Rønne-Hansen:}

Responding Göran Andersson

1) The primary aim of the work presented in the paper is to extend in an easy and practical way the usability of the traditional transient stability method. The author is fully aware of the fact that the primary use of such programs is in power system planning and protection. And for this purpose there is no need for extending the representation of unsymmetric conditions. However, now and then complicated faults appear in the network causing unexpected reactions in the system. These incidents call for a wider range of possibilities for representing not only a very complicated fault or combination of faults - but also the ability to analyze in detail those parts of the network or its components which behave inexplicably. Of course, very detailed transient programs can be used for this purpose, but the computational burden is much heavier when using such program. And the data needed in these programs might not be versatile for the purpose. To the authors opinion the method presented instead of representing "brute force" rather can be considered a way to allow the user to concentrate on the essential problems in stead of overwhelming him with lots of useless numbers. And hence help him to gain the insight which - according to Richard Hamming - is the very purpose of computing. To complete this remark it should be stressed that by using the nodal admittance matrix when describing the unsymmetry it is hoped that the method can be understood and made practical for users without too deep theoretical knowledge.

2) The original aim was - as already stated - to allow modelling of unsymmetries which previously were extremely difficult to represent when considered from the positive sequence network. However, the method may have further and equally important applications since it makes it possible to switch freely between phase quantities and positive sequence representation in the same network. When dealing with heavy unsymmetries in a large network the immediate neighbourhood of the fault can be modelled by full three phase representation, whereas more distant parts of the system can be represented by the positive sequence scheme. Even the negative and when necessary - zero sequence part for the entire network is taken into account, but only represented by the boundary nodes connecting the three phase models with the major part of the network modelled by means of the positive sequence data. This could just as well be obtained by means of symmetrical components alone, but the application of three phase models leaves the user more freedom to model the components. Even e.g. nonlinear elements can be included in the system in a detailed way. Including the conversion circuit as a mathematical "component" in the program the conventional transient stability program thus can be applied in a more flexible way. The number of extra nodes made necessary by the conversion between symmetrical components and phase quantities may seem big. But no excessive nodes exist when considering the minimum negative and zero sequence network equivalents.

Responding Magnus Akke:

It is correct that including thee phase representation in parts of the network in which the negative sequence quantities are essenticl a more accurate perception of the damping can be achieved. Also the influence on the generators can be considered in a precise manner. As already stated above the user has the full freedom to decide which parts of the network that should be modelled in the greater detail following a threephase model. The extent of the three phase modelling must depend upon the judgement of the user when evaluating the actual problem. Whether or not the enhanced method actually will change the critical clearing time enough to cause actual adjustments of relay settings the author doubts, but considering negative sequence currents - and for that sake zero sequence quantities as well - a more accurate representation is made possible.

Manuscript received November 14, 1996. 